\title{
¿QUÉ ES UN CONTEXTO PARA LAS TEORÍAS COGNITIVAS DEL RAZONAMIENTO?
}

\author{
JONATAN GARCÍA CAMPOS \\ Universidad Juárez del Estado de Durango \\ Instituto de Ciencias Sociales \\ jongarcam@yahoo.com.mx \\ SAÚl SARABIA López \\ Universidad Nacional Autónoma de México \\ Posgrado en Filosofía de la Ciencia \\ saulsarabia24@gmail.com
}

RESUMEN: El artículo tiene como propósito analizar la noción de contexto en algunas de las teorías más importantes en la psicología cognitiva. Argumentaremos que la noción de contexto puede hacer referencia a al menos cinco ideas: la familiaridad del contenido, el formato del problema, la perspectiva del sujeto, la estructura lógica distinta y el ambiente. Indagaremos las relaciones posibles entre dichas nociones de contexto y esbozaremos respuestas a dos preguntas que pueden plantearse al análisis presentado, esto es, cuál es la razón por la que se tienen diferentes nociones de contexto en la psicología cognitiva, y cuál podría ser la consecuencia en los debates sobre razonamiento y racionalidad si se distinguen distintas nociones de contexto.

PALABRAS CLAVE: familiaridad, perspectiva, ambiente, tarea de selección, falacia de la conjunción

SUMMARY: The purpose of this article is to analyze the notion of context in some of the most important theories of cognitive psychology. We shall argue that the notion of context can make reference to at least five different ideas: familiarity of the content, problem format, subject's perspective, different logical structure, and environment. We shall investigate what relationships between the notions of context might be, and we shall draw to an answer two questions that can be raised to our analysis, i.e., why there are different notions of context in cognitive psychology, and what the consequences would be to reasoning and rationality debates if we distinguish different notions of context.

KEY WORDS: familiarity, perspective, environment, selection task, conjunction fallacy

En un famoso debate entre dos de los grupos más importantes en la psicología cognitiva del razonamiento (la tradición de heurística y sesgo, y el grupo ABC) encontramos una acusación común, a saber, que la postura rival no toma en cuenta el contexto en el cual los seres humanos razonamos. En este debate, recogido en parte por la revista Psychological Review, Gigerenzer acusa explícitamente a Kahneman 
y Tversky de no tener en cuenta el contexto en el estudio del razonamiento. Gigerenzer sostiene que, en la tradición de heurística y sesgo, "los juicios de lo que cuenta como un razonamiento adecuado pueden ser independientes [...] del contexto" (1996, pp. 592-593). Ante esta acusación, los defensores de la tradición de heurística y sesgo afirman:

Quizá la más seria malinterpretación de su parte [refiriéndose a Gigerenzer] se relaciona con que los juicios heurísticos son "independientes... del contexto"... Nada puede estar más alejado de la verdad. El reconocimiento de que diferentes formas de presentar el mismo problema [...] pueden dar lugar a diferentes procesos mentales ha sido una constante de nuestro acercamiento... (Kahneman y Tversky 1996, p. 583, las cursivas son nuestras.)

Así, dos teorías psicológicas en pugna consideran que toman en cuenta el contexto en el que el sujeto razona y al mismo tiempo afirman que su respectiva teoría rival no lo hace. Esto causa que nos preguntemos si estas posturas psicológicas están hablando de lo mismo cuando hacen uso de la noción de contexto. Sin embargo, dar respuesta a la pregunta anterior es difícil, puesto que ni Kahneman y Tversky, por un lado, ni Gigerenzer y su grupo de investigación, por otro, explican qué se debe entender por contexto, o cómo diferenciar entre diferentes contextos. De hecho, como se expondrá en este trabajo, es posible señalar que esta carencia no es exclusiva de la tradición de heurística y sesgo ni del grupo $\mathrm{ABC}$, pues otros enfoques en la psicología cognitiva también carecen de tal análisis.

El trabajo que aquí presentamos tiene como objetivo responder a la pregunta de qué es un contexto en algunas teorías de la psicología cognitiva del razonamiento. Dada la variedad de propuestas para explicar la competencia de razonamiento, nos centraremos en algunas de las tareas de razonamiento más importantes, como la tarea de selección de tarjetas y la denominada falacia de la conjunción, así como en algunas teorías sobre el razonamiento. Mostraremos que a pesar de que el término contexto se encuentra en la literatura, con él se hace referencia a distintas ideas y, como argumentaremos, no existe una descripción o un análisis claro de qué se debe entender por ese término. Así, a lo largo del trabajo caracterizaremos distintos sentidos en que se ha usado la noción de contexto en algunos de los trabajos de la psicología cognitiva.

Aquí propondremos que según la noción de contexto se hace referencia a distintas ideas; entre ellas están: 1) la familiaridad o la 
falta de familiaridad de los problemas (Cosmides y Tooby 1992); 2) el formato con que se formula un problema (Cosmides y Tooby 1996; Gigerenzer y Hoffrage 1995); 3) la perspectiva del sujeto en relación con la tarea por resolver (Cosmides y Tooby 1992); 4) la estructura lógica que se usa en una tarea de razonamiento (Fodor 2000; Buller 2005; Buller et al. 2005); y 5) la noción de ambiente (Gigerenzer y Sturm 2012; Arnau et al. 2013). Así, en este trabajo trataremos de mostrar, en primer lugar, la existencia de estas ideas vinculadas con la noción de contexto y, en segundo lugar, haremos un esbozo de las posibles relaciones entre ellas. Cabe señalar que no nos pronunciaremos a favor de una noción particular de contexto, es decir, no intentaremos mostrar cuál de las anteriores nociones es la mejor, sino que nos limitaremos a hacer patente cómo las distintas teorías se han comprometido con diferentes nociones y que muchas veces, dentro de una misma teoría, se usa la noción de contexto de distintas maneras. Aunque el propósito de este trabajo no es explicar cómo es que los psicólogos cognitivos llegan a tener nociones de contexto distintas, sí deseamos esbozar al final qué podría dar cuenta de esas diferencias, así como mostrar de modo general por qué puede ser relevante tomar en cuenta esas diferencias para el tema de razonamiento y racionalidad en la psicología cognitiva.

\section{El contexto como la familiaridad de un problema}

Stein (1996, p. 83) al explicar una de las tareas más conocidas sobre el razonamiento, la de selección de tarjetas, sostiene que en ésta se empezó rápidamente a señalar que el contexto es importante para la resolución de los problemas cognitivos. Sin embargo, tan pronto como emplea dicho término, comenta que lo anterior es el caso porque algunos psicólogos se dieron cuenta de que el contenido familiar o no familiar (o abstracto, como a veces se denomina) de la tarea de selección de tarjetas parece tener un efecto en la ejecución del razonamiento. La tarea abstracta se presenta como sigue:

Todas las cartas que verás a continuación tienen de un lado un número y del otro una letra. Tu trabajo es determinar cuáles de estas cartas tienes que voltear para probar la verdad de la oración:

"Si hay una vocal en un lado de la carta, entonces hay un número par en el otro lado"
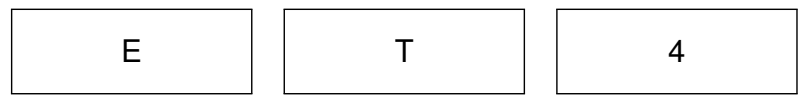

7 
Wason (1966) y otros psicólogos descubrieron que por lo común los seres humanos no respondemos correctamente a este tipo de tareas, pues aun cuando la respuesta correcta a este experimento es voltear las tarjetas E y 7, la mayoría de los sujetos (75\%) sólo consideran que se debe voltear la carta E, o las cartas E y 4. Sin embargo, a diferencia de esa tarea abstracta, la versión familiar de la tarea de selección de tarjetas tuvo resultados distintos. He aquí un ejemplo:

Se les pide a los sujetos que imaginen que cada tarjeta representa de un lado un viaje hecho por el experimentador y del otro lado un medio de transporte. Se les pide a los sujetos probar la validez de una regla como la siguiente:

"Si voy a Nueva York, entonces viajo en tren"
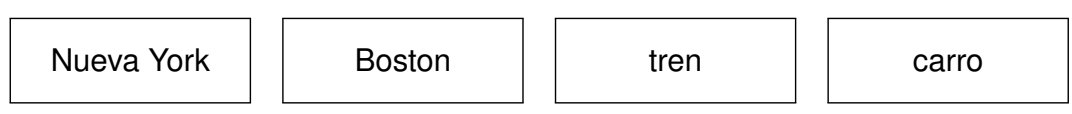

Casi dos tercios de los sujetos seleccionaron correctamente las tarjetas "Nueva York" y "carro", esto es, consideraron que estas tarjetas debían examinarse. Esto es dramáticamente mejor que el menos del $10 \%$ que seleccionó las cartas correctas en la versión abstracta de la tarea que hemos presentado.

Dadas estas versiones de la tarea de selección, parece que es fácil distinguir entre tareas familiares y no familiares. ¿Qué significa exactamente que algo sea familiar? Los psicólogos cognitivos no explican qué es la familiaridad o la abstracción de un problema y simplemente consideran que estos términos son lo suficientemente claros. Sin embargo, nosotros pensamos que hay una manera general de entender que un problema es familiar, i.e., que el contenido que define el problema es familiar. ${ }^{1}$ Por contenido entendemos aquí el tipo de objetos que se encuentran en las tarjetas (números o medios de transporte-ciudad, en los ejemplos usados). Para poder usar la noción de contenido de manera más general, podríamos decir que el contenido de una tarea de razonamiento hace referencia a los objetos o términos usados en el problema de razonamiento particular.

La familiaridad es importante debido a que algunas teorías han defendido que la ejecución adecuada de una tarea de razonamiento

${ }^{1}$ De hecho, en distintos trabajos los psicólogos evolucionistas (Cosmides y Tooby 1992, 2005) aplican el adjetivo "familiar" o el sustantivo "familiaridad" al contenido, la relación, la regla, la situación, el problema y los términos. Aunque, como veremos más adelante, cuando hablan de familiaridad con respecto a la tarea de selección de tarjetas, se centran en el contenido. 
depende de la familiaridad de dicha tarea. Por ejemplo, en las dos tareas de selección antes presentadas se considera que la diferencia en la ejecución se debe simplemente a la familiaridad del contenido. Sin embargo, los psicólogos evolucionistas han tratado de poner en duda lo anterior, en particular (Cosmides y Tooby 1992) creen que hay evidencia de que problemas no familiares pueden ofrecer buenos resultados, mientras que problemas familiares pueden ofrecer malos resultados, lo que, de acuerdo con ellos, demuestra que la familiaridad no explica un mejor desempeño en las tareas de razonamiento. Una de las tareas de selección de tarjetas en donde presentan esto es la siguiente:

Los miembros del pueblo Kalume consideran que la raíz de cassava es un afrodisiaco muy poderoso y, por ello, todos desean comerla. Además, esta raíz es muy sabrosa y nutritiva, y el que la consume aumenta su atractivo físico. Otro alimento característico de este pueblo son las nueces de molo, que no provocan que las mujeres se sientan atraídas físicamente por el hombre que las come.

El problema es que la raíz de cassava es bastante escasa y hay que racionarla. Razón por la que sólo se permite consumirla a los hombres casados. Es fácil identificar a un kalume casado, ya que cuando un kalume contrae matrimonio se tatúa el rostro. Los líderes del pueblo Kalume condenan las relaciones sexuales entre las personas no casadas.

Las siguientes tarjetas contienen información de 4 hombres, un lado de la tarjeta indica qué es lo que el hombre está comiendo y el otro lado indica si tiene o no un tatuaje en su cara. Indica solamente las tarjetas que tienes que voltear para ver si alguno de los hombres kalume está rompiendo la regla:

"Si un hombre come raíz cassava, entonces debe tener un tatuaje en su cara"
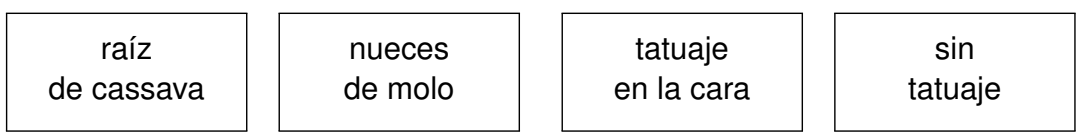

En esta versión, alrededor del $75 \%$ de los sujetos resolvieron correctamente la tarea. Este experimento de la raíz de cassava demuestra, según los psicólogos evolucionistas, que los sujetos pueden tener un buen desempeño para resolver tareas de selección no familiares. No así experimentos familiares como el que Stein (1996) presentó:

En un experimento, se presentó a los sujetos un mazo de tarjetas referidas a lo que el experimentador comió y tomó en una comida particular. Después se estableció una regla como la siguiente: 
"Si como carne de puerco, entonces tomo vino tinto"

Posteriormente se mostraron cuatro tarjetas a los sujetos como las siguientes:
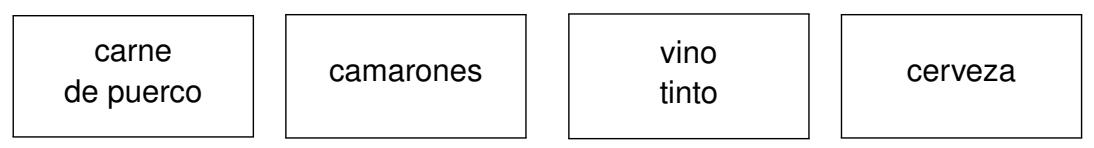

Según Stein (1996), los sujetos tuvieron el mismo desempeño en la selección de tarjetas de la comida y bebida que en la tarea de selección de tarjetas abstracta que presentamos anteriormente, lo cual, como ya señalamos, lo usan los psicólogos evolucionistas para mostrar que la familiaridad de los problemas no explica la mejora en las tareas de razonamiento. ${ }^{2}$

En suma, queremos señalar que en este caso el término contexto se reduce a la familiaridad del problema presentado en una prueba, i.e., se dice vagamente que un contexto es familiar o no es familiar haciendo referencia al contenido del problema. En la tarea de selección de tarjetas, la familiaridad del problema recae, según vemos, en si el contenido de dicho problema es familiar o no; por ejemplo, si se trata de comidas y bebidas o medios de transporte y ciudades. ${ }^{3}$ ¿Es ésta la única manera en que se ha usado la noción de contexto en la psicología cognitiva del razonamiento? Como veremos en la siguiente sección, ésta no es la única manera en la que se ha entendido qué es un contexto.

\section{El contexto como el formato en que se plantea un problema}

Junto con la tarea de selección de tarjetas, uno de los problemas más intensamente estudiados en torno al razonamiento es en relación con los juicios sobre la probabilidad, en particular, un experimento conocido coloquialmente como "Linda, la cajera feminista". El experimento es el siguiente:

${ }^{2}$ La idea que defienden los psicólogos evolucionistas para explicar esta diferencia se debe al módulo de detección de tramposos, esto es, un mecanismo cognitivo producto de la evolución que permite detectar a aquellos que reciben un beneficio en un contrato social sin pagar su costo (Cosmides y Tooby 1992).

${ }^{3}$ Ciertamente hablar de familiaridad con el contenido puede resultar vago en un sentido general, pero dentro de la tarea de selección podría entenderse como los términos que aparecen en las tarjetas que se deben voltear, que además aparecen en el condicional que se va a probar. Algunos psicólogos cognitivos, como hemos visto, han pensado que la ejecución de los sujetos cambia si las tarjetas contienen letras y números, en vez de medios de transporte y ciudades. 
Linda tiene 31 años de edad, es soltera, inteligente y muy brillante. Estudió filosofía y como estudiante estuvo profundamente preocupada por los problemas de discriminación y justicia social. Linda participó también en manifestaciones antinucleares.

De los siguientes enunciados indica qué es más probable:

1. Linda es maestra de primaria.

2. Linda es cajera en un banco.

3. Linda es cajera en un banco y es activista de movimientos feministas.

En un grupo de personas sin ningún estudio en probabilidad y estadística, 89\% consideraron que el enunciado (3) es más probable que el enunciado (2). Este resultado parece señalar que los sujetos del experimento cometen la falacia de la conjunción al sostener que un evento compuesto ("Linda es cajera y feminista") es más probable que un evento simple ("Linda es cajera"). ${ }^{4}$ El resultado es similar en sujetos con estudios en matemáticas, alrededor del $80 \%$ cometen la misma falacia (Piatelli-Palmarini 2005, pp. 73 y 115). Lo anterior ha hecho que se defienda que los sujetos del experimento no cuentan con la competencia de razonamiento adecuada.

Sin embargo, tanto los psicólogos evolucionistas como el grupo ABC ponen en duda la interpretación de este problema. Según ellos, en ciertos contextos es posible mostrar cómo los seres humanos sí tienen la competencia adecuada (Gigerenzer 1991, pp. 92 y 108; Cosmides y Tooby 1996, pp. 67 y 69; Hertwig y Gigerenzer 1999, pp. 276, 278, 279, 285 y 300). La manera en que los psicólogos evolucionistas y Gigerenzer y su grupo presentan este caso es como un "formato del problema". Veamos.

Partiendo de ciertos supuestos evolucionistas acerca del tipo de problemas a los que los ancestros humanos pudieron enfrentarse, Cosmides y Tooby, así como Gigerenzer y su grupo, proponen que seguramente la mente humana seleccionó un tipo de mecanismo que permite procesar información que es proporcionada en formato de frecuencias y hacer generalizaciones a partir de éstas. A esta hipótesis

${ }^{4}$ El principio que se estaría violando parte de la teoría de la probabilidad y comúnmente se formula como sigue: $P(A \& B) \leq P(A)$. Detrás de la violación de una regla de razonamiento se está partiendo de una visión estándar de la racionalidad, según la cual "ser racional es razonar conforme a los principios de razonamiento que están fundados en las reglas de la lógica, la teoría de la probabilidad u otras teorías formales" (Stein 1996, p. 4). 
se le conoce como la hipótesis frecuentista (Cosmides y Tooby 1996; Gigerenzer y Hoffrage 1995; Hertwig y Gigerenzer 1999; Samuels et al. 2004). En sus propias palabras: "La hipótesis [frecuentista sostiene] que parte de nuestros mecanismos de razonamiento inductivo incorporan aspectos del cálculo de probabilidad, pero están diseñados para tomar frecuencias como insumos y producir frecuencias como resultados" (Cosmides y Tooby 1996, p. 5). Según los defensores de la hipótesis frecuentista, si la mente cuenta con mecanismos que procesan y dan como resultado información en formato de frecuencias, entonces, en los contextos constituidos por estos formatos, dichos mecanismos ofrecerán una respuesta normativamente adecuada. ${ }^{5}$ De hecho, su trabajo experimental parece mostrar lo anterior. ${ }^{6}$ Se presenta el mismo experimento de "Linda, la cajera feminista" y en él parece haber una ejecución diferente. La versión frecuentista del experimento es la siguiente:

Linda tiene 31 años de edad, es soltera, inteligente y muy brillante. Estudió filosofía y como estudiante estuvo profundamente preocupada por los problemas de discriminación y justicia social; participó también en manifestaciones antinucleares.

Hay 100 personas que cumplen con la descripción arriba presentada. ¿Cuántas de ellas son:

1. maestras de primaria?

2. cajeras?

3. feministas y cajeras en un banco?

Según los psicólogos evolucionistas, en una réplica del experimento de Kahneman y Tversky, usando la formulación original que presentamos anteriormente, el $89 \%$ de los sujetos consideró que la opción "feministas y cajeras de un banco" era más probable que la opción "cajeras". No obstante, en la versión frecuentista del mismo

${ }^{5}$ La distinción entre probabilidades frecuentistas y subjetivistas que presentamos aquí sigue de cerca el debate que han tenido los psicólogos cognitivos aquí expuestos, no pretendemos pronunciarnos sobre la validez de esta distinción, sino que la tomamos como punto de partida para mostrar cómo se relaciona con la noción de contexto.

${ }^{6}$ En este trabajo nos centramos en el problema de "Linda, la cajera feminista"; sin embargo, éste no es el único experimento que ha sido reinterpretado por los defensores de la hipótesis frecuentista; por ejemplo, ellos consideran que los sesgos en relación con el exceso de confianza o el rechazo de la razón de base también desaparecen (Cosmides y Tooby 1996, p. 62). 
experimento, según estos mismos psicólogos, solamente el $22 \%$ de los sujetos consideraron que la opción "feministas y cajeras de un banco" era más probable que la opción "cajeras".

Si bien resulta interesante que los resultados entre dos versiones de un mismo problema son significativamente distintos, nos interesa dirigir la atención aquí a que las dos versiones cuentan con el mismo contenido (las dos se refieren a la misma mujer y a las mismas actividades laborales) y, sin embargo, el formato en que se presentan es distinto. En la primera versión del experimento revisado en esta sección se tiene un formato subjetivista, pues se hace una pregunta por la probabilidad subjetiva de un evento singular. En la segunda versión, en cambio, se está frente a un formato frecuentista, pues la pregunta está planteada por la frecuencia de un evento dentro de una clase particular. Los dos formatos son maneras de representar información probabilística que es matemáticamente equivalente, pero que puede ser procesada o no con los algoritmos adecuados (Gigerenzer y Hoffrage 1995). El ejemplo que a veces se usa para ilustrar los diferentes formatos hace referencia a los distintos formatos en que se presentan los números (Cosmides y Tooby 1996, p. 18). ${ }^{7}$ Los números pueden ser representados en diferentes sistemas (romano, arábigo, binario, etc.). Esas representaciones pueden ser convertidas o trasladadas de un sistema a otro y en ese sentido pueden ser matemáticamente equivalentes. No obstante, la forma de representación, según los psicólogos evolucionistas (Cosmides y Tooby 1996), puede marcar una gran diferencia en el algoritmo que hace la multiplicación. Por ejemplo, una calculadora de bolsillo que ha sido creada para recibir números arábigos como insumos fallaría en realizar un cálculo si le introdujéramos números binarios, aunque dicha calculadora funcione correctamente. De igual manera, estos psicólogos cognitivos sugieren que las personas cuentan con al menos un mecanismo cognitivo diseñado para hacer cálculos probabilísticos en formato de frecuencias. Así como una calculadora fue hecha para operar solamente con números arábigos como insumos, así también la mente del ser humano fue diseñada para realizar los cálculos probabilísticos con formatos de frecuencias como insumos.

\footnotetext{
${ }^{7}$ Por supuesto, se trata del ejemplo de los teóricos que defienden esta postura y no que nosotros consideremos que la relación que guardan los distintos sistemas numéricos (por ejemplo, que sean matemáticamente isomórficos) sea idéntica a la relación que se guarda entre las probabilidades subjetivas y objetivas.
} 


\section{El contexto como perspectiva}

Hasta aquí hemos señalado que el término contexto se ha equiparado con la familiaridad del problema y con el formato de un problema, pero estos modos no son los únicos sentidos en que se ha hecho uso de este término. Volviendo de nuevo a la tarea de selección de tarjetas, Gigerenzer y su grupo han descubierto que la buena ejecución de un problema de razonamiento depende de la perspectiva que un sujeto tiene que tomar en la tarea en cuestión (Samuels et al. 2004). Lo interesante es que cuando hacen referencia a la perspectiva que toma el sujeto que razona, nuevamente usan el término contexto (Gigerenzer y Hug 1992, p. 130). Veamos.

En una versión de la tarea de selección de tarjetas llamada "cambio de perspectiva", Gigerenzer y Hug (1992) dieron a dos grupos una tarea de selección de tarjetas en la que deberían buscar violaciones de las normas del contrato social como: "Si un empleado recibe una pensión, entonces ese empleado debe haber trabajado para la empresa durante al menos 10 años". La única diferencia entre los dos grupos fue que a un grupo se le dijo "Tú eres el empleador", mientras que al otro grupo se le dijo "Tú eres el empleado". Las tarjetas presentadas fueron las siguientes:
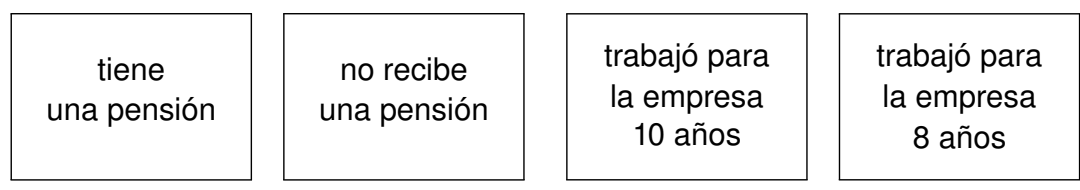

En teoría del contrato social, lo que cuenta como hacer trampa o la violación de un contrato depende muchas veces de la perspectiva que uno tenga dentro del contrato (pensemos, por ejemplo, en un contrato de compraventa en el que el vendedor y el comprador tienen distintos derechos y obligaciones) (Cosmides y Tooby 1996). En el caso de la tarea de selección recién presentado, proveer una pensión es un costo que tiene que pagar el empleador para beneficiar al empleado, mientras que trabajar por 10 años o más es un costo que el empleado tiene que pagar para beneficiar al empleador. De esta manera, el evento "Si un empleado recibe una pensión" es considerado un costo o un beneficio dependiendo de si se toma la perspectiva del empleador o del empleado, aun cuando se trate del mismo antecedente del condicional. Del mismo modo, el consecuente "debe haber trabajado para la empresa al menos 10 años" puede verse como un costo desde el punto de vista del empleado, pero como un 
beneficio en la perspectiva del empleador, aun cuando se trate del mismo consecuente. En suma:

- desde la perspectiva del empleador se tiene que "Si un empleado recibe una pensión, entonces ese empleado debe haber trabajado para la empresa durante al menos 10 años", se lee como "si tengo un costo (pagar una pensión), entonces tengo un beneficio (tengo a alguien trabajando)";

- desde la perspectiva del empleado se tiene que "Si un empleado recibe una pensión, entonces ese empleado debe haber trabajado para la empresa durante al menos 10 años", se lee como "si tengo un beneficio (tengo una pensión), entonces tengo un costo (haber trabajado en esa empresa por varios años)".

Lo interesante de estas tareas de selección es que nos encontramos frente al mismo contenido. De hecho, en los dos casos el contenido es familiar; sin embargo, la ejecución cambia porque la perspectiva tomada por el sujeto razonador es distinta.

El número de perspectivas que puede tener un problema de razonamiento depende del problema particular que esté enfrentando el sujeto. En el ejemplo anterior de tarea de selección de tarjetas existen dos diferentes perspectivas, esto es, la del empleado y la del empleador, pero es posible que otras tareas de razonamiento puedan tener mayor número de perspectivas. Los psicólogos evolucionistas han usado los resultados de estos experimentos para defender tesis muy particulares que no son de interés en este trabajo, ${ }^{8}$ pero en este aspecto Cosmides y Tooby señalan que:

La definición de trampa también depende del punto de vista que el agente está tomando. La perspectiva importa por el objeto, acción o estado de cosas que desde una parte se ve como un beneficio puede ser visto como un requisito desde la otra parte. El sistema necesita ser capaz de computar las representaciones costo-beneficio desde la perspectiva de un participante y definir la trampa a partir de esa perspectiva relativa. (2005, p. 593)

Si lo hasta aquí señalado es correcto, la perspectiva parece representar otra manera de ver un contexto en las tareas de razonamiento cognitivo.

${ }^{8}$ Véase la nota 2 en este texto. 


\section{El contexto como estructura lógica distinta}

En las secciones 1 y 3 del trabajo hemos expuesto diferentes versiones de la tarea de selección de tarjetas. Los diferentes resultados en la ejecución de dichas tareas han sido usados por distintos teóricos para señalar que el cambio de contexto es importante para la solución de una tarea de razonamiento. Distintos teóricos (Cosmides y Tooby 2005; Stein 1996; Samuels et al. 2004) han sostenido que lo que tienen en común esas tareas de selección es una misma estructura lógica, pues en todas ellas se pide probar la verdad de un enunciado del tipo "si A, entonces B". Por ese motivo, se ha buscado la explicación de las diferencias en la ejecución de la tarea de selección de tarjetas no en aspectos lógicos, sino en el contenido del problema o en la perspectiva de los individuos ante éste.

A diferencia de estas explicaciones, críticos de los psicólogos evolucionistas, en especial Fodor (2000) y Buller (2005, véase también Buller et al. 2005), han señalado que lo que explica la diferencia entre la ejecución en las tareas de selección se debe a que tienen una estructura lógica distinta. En particular, estos autores sostienen que en la tarea de selección abstracta presentada en la sección 1 (aquella con el condicional "Si hay una vocal en un lado de la carta, entonces hay un número par en el otro lado"), el condicional es de tipo indicativo. Mientras que en las tareas de selección con contenido social (por ejemplo, aquel con el condicional "Si un hombre come raíz cassava, entonces debe tener un tatuaje en su cara"), el condicional es de tipo deóntico. Así, las tareas de selección abstracta y aquellas con contenido familiar de tipo social, según estos autores, sólo parecen tener la misma estructura "si A, entonces B", pero en realidad hacen referencia a un condicional indicativo o a un condicional deóntico.

Según Buller (2005), en los condicionales de tipo indicativo "si A, entonces B" la verdad de la oración B es condicional o depende de la verdad de la oración A. Los condicionales indicativos se usan para hacer afirmaciones sobre hechos. En cambio, los condicionales deónticos "si A, entonces B" lo hacen para imponer obligaciones. En lugar de hacer afirmaciones sobre la verdad de una oración B a partir de la verdad de otra oración A, los condicionales deónticos crean una obligación a partir de la afirmación de una oración. En el caso de "Si un hombre come raíz cassava, entonces debe tener un tatuaje en su cara", se crea la obligación o recomendación de "tener un tatuaje en la cara" a partir de "comer raíz de cassava". De hecho, Buller et al. (2005, p. 509) distinguen entre dos tipos de condicionales deónticos, viz., obligaciones y recomendaciones. Más precisamente, sostener "es 
obligatorio que $\mathrm{P}$ " implica "está prohibido que no-P", mientras que sostener "es recomendable que $\mathrm{P}$ " implica "no está prohibido que P".

La idea de Buller es que el resultado de la ejecución adecuada en tareas de selección se debe a que el condicional es indicativo o es deóntico, debido a que "las tareas de selección que producen un nivel de ejecución alto implican condicionales deónticos, mientras que las tareas de selección que producen un nivel de ejecución bajo implican condicionales indicativos" (Buller 2005, p. 182). Lo interesante para los propósitos de este trabajo es que cuando los defensores de esta propuesta presentan esta diferencia en los condicionales, afirman que los tipos de condicionales dan lugar a, o están inmersos en dos contextos distintos. Buller afirma que una manera de entender por qué los sujetos no responden de manera adecuada a la tarea de selección se debe a que:

Los condicionales indicativos incorporan conexiones arbitrarias entre el antecedente y el consecuente, y las historias en las que los condicionales están incrustados proveen información contextual insuficiente para hacer que la conexión sea sensata $[\ldots]$; debido a que esta información está ausente, los sujetos no saben cuál es el tipo lógico de la oración condicional que tienen enfrente, y en consecuencia son incapaces de ejecutar las tareas lógicas con respecto a ese condicional. (2005, p. 180, las cursivas son nuestras.)

Así, Buller parece afirmar que el condicional indicativo suele ser arbitrario y, además, está acompañado de una historia que no permite a los sujetos darse cuenta del tipo de condicional que "tienen enfrente". De este modo, el contexto parecería formarse tanto por el condicional indicativo como por una "historia". Aun cuando no se define qué es una "historia", ésta puede entenderse como el contenido o la información previa. Dado que estos teóricos ponen el énfasis en la naturaleza del conectivo lógico y no en la "historia" (Buller et al. 2005, p. 509), consideramos que la parte central de la noción de contexto aquí revisada se encuentra en una estructura lógica distinta, en particular, en el conectivo lógico, ya sea deóntico o indicativo.

\section{El contexto como ambiente}

A manera de introducción y para motivar el estudio de la noción de contexto, hemos iniciado este trabajo con un debate clásico en la psicología cognitiva del razonamiento, a saber, entre la posición defendida por la tradición de heurística y sesgo y aquella defendida 
por el grupo ABC. Como hemos mencionado, ambos grupos de psicólogos acusan al grupo rival de no tener en cuenta el contexto en el que los sujetos razonan (aunque supuestamente su propia postura sí lo hace), ${ }^{9}$ pero no explican qué se debe entender por éste (Gigerenzer 1996, pp. 592-593; Kahneman y Tversky 1996, p. 583).

Una posible manera de entender "contexto" en este debate, al menos con relación al grupo $\mathrm{ABC}$, es recurrir a la idea que han desarrollado Gigerenzer y su grupo en cuanto al ambiente. Esta noción de ambiente según Gigerenzer, como se verá más adelante, está relacionada con la noción de contexto debido a que muchas veces ellos ven el cambio de ambiente como un cambio de formato subjetivista a frecuentista, o viceversa, tal y como se presenta en su discusión con Kahneman y Tversky. Si algunas veces Gigerenzer y el ABC llaman a esto cambio de formato y lo identifican como ambiente, entonces es posible que ellos vean en general el ambiente como una manera de entender el contexto.

Siguiendo la metáfora de las tijeras que originalmente usó Herbert Simon, los defensores de la racionalidad ecológica señalan que la conducta racional está moldeada por dos navajas de una tijera: el ambiente y la capacidad computacional del individuo. En otras palabras, Gigerenzer y su grupo afirman que la racionalidad de una capacidad mental que procesa información (en particular, ellos han defendido la existencia de heurísticas rápidas y frugales) depende del buen ajuste que tenga con una estructura ambiental particular (Gigerenzer et al. 1999). Así, su programa busca entender la interacción entre las capacidades mentales y el ambiente. ¿Qué es lo que Gigerenzer y su grupo entienden por ambiente? Los defensores del grupo ABC, que muchas veces han denominado a su propuesta racionalidad ecológica, ${ }^{10}$ no han aclarado cómo se debe entender esta noción. Algunas veces acompañan la noción de ambiente con los adjetivos "físico" y "social"; otras veces la idea de "la estructura informacional" antecede a la mención del ambiente físico y social. Teniendo en cuenta lo visto en las secciones anteriores, ¿puede la estructura del ambiente producir los formatos representacionales o la naturaleza del tipo de conectivos que se usan, o todo lo anterior

\footnotetext{
${ }^{9}$ Los psicólogos evolucionistas, al igual que las dos propuestas del debate aquí apuntado, también afirman que toman en cuenta el contexto. Ellos afirman, por ejemplo, que "los datos sobre el razonamiento inductivo indican que no contamos con procedimientos de razonamiento inductivo independientes [...] de contexto" (Cosmides y Tooby 1991, p. 499).

${ }^{10} \mathrm{El}$ adjetivo "ecológico" es usado precisamente por la importancia que en esta teoría tiene el ambiente en el que el sujeto razona.
} 
junto? Nosotros no tenemos claro qué es lo que exactamente señala Gigerenzer con la estructura informacional de los ambientes físicos y sociales, pues esa estructura puede hacer referencia a varias ideas que no son equivalentes; por ejemplo, no es lo mismo el formato en que se presenta un problema de razonamiento (que podría ser subjetivo o frecuentista en el caso de un razonamiento probabilístico), que el tipo de condicional de un problema (que podría ser deóntico o indicativo).

Pero la ambigüedad de la noción de ambiente no termina aquí. Haciendo una crítica a la idea de racionalidad ecológica que Gigerenzer defiende, Stanovich y West (2003) señalan directamente que otro punto poco claro en su propuesta es si el ambiente hace referencia al ambiente de adaptación evolutiva (AAE) o al ambiente actual. ${ }^{11}$ En el primer caso, el ambiente estaría constituido por las características reales del pasado que ejercieron presión selectiva en la mente de nuestros ancestros. Esta idea estaría reforzada por la visión del grupo $\mathrm{ABC}$ de que los mecanismos de los seres humanos son parte de una caja de herramientas adaptativas con una estructura evolutiva y que algunas de ellas son compartidas con otros animales (Gigerenzer y Sturm 2012, p. 248). Pero, por otro lado, la idea del ambiente actual se ve reforzada porque el grupo ABC (Gigerenzer y Sturm 2012 , p. 243) no sólo pretende describir cómo la gente razona, sino también identificar los ambientes específicos en los cuales los sujetos contemporáneos pueden, de hecho, hacer inferencias.

Frente a esta ambigüedad en cuanto a si la noción de ambiente hace referencia a la estructura informacional del ambiente social o físico, que además puede ser entendido como el $\mathrm{AAE}^{12}$ o el ambiente actual, podemos traer a colación el trabajo de Arnau et al. (2013) que presenta una manera de entender el ambiente para determinar en qué medida la propuesta del grupo ABC es cercana a las posturas situadas en la cognición. Arnau et al. (2013) proponen tres grandes maneras de entender qué es el ambiente a partir de sus aspectos descriptivo, normativo y prescriptivo. ${ }^{13} \mathrm{El}$ aspecto descriptivo en los estudios de razonamiento se relaciona con la descripción de cómo los seres

${ }^{11}$ Con esto no queremos sostener que la teoría dual de Stanovich y West iguale la noción de contexto con la noción de ambiente; simplemente señalamos cómo ellos han apuntado la ambigüedad de la noción de ambiente de Gigerenzer y su grupo.

${ }^{12}$ De hecho, es posible problematizar todavía más la idea de ambiente como AAE si tenemos en cuenta las observaciones que han hecho Buller (2005), Bolhuis et al. (2011) y Chiappe y Gardner (2011) sobre la inestabilidad del AAE y cómo no se puede hablar de un único ambiente para nuestros ancestros cazadores recolectores. Si este tipo de observaciones son adecuadas, hacer referencia al AAE como un único ambiente, sería en el mejor de los casos una sobresimplificación.

${ }^{13}$ Estas tres maneras de entender el ambiente están relacionadas con la totalidad 
humanos razonamos. El aspecto normativo con la manera en que debemos razonar. Mientras que el aspecto prescriptivo se relaciona con las estrategias de razonamiento particulares que un sujeto debe seguir. La propuesta defendida por la racionalidad ecológica intenta cubrir estos aspectos (Gigerenzer y Sturm 2012) y, según Arnau et al. (2013), la noción de ambiente está presente en cada uno de ellos. Veamos.

- Según Arnau et al. (2013), una de las maneras de ver el ambiente es como el ambiente físico. De acuerdo con estos autores, algunos ejemplos de heurísticas requieren que el agente esté en contacto con el ambiente físico relevante. Además, el agente confía en la manipulación del ambiente para solucionar los problemas. "En la medida en que alteran la estructura física e informacional del ambiente, esos procesos conducen al agente a estar más cerca de la solución de un problema; así, son parte del procesamiento del problema del agente" (Arnau et al. 2013, p. 9). Un ejemplo de ambiente físico y su relación con las heurísticas es el que usan Gigerenzer y su grupo para explicar cómo un jugador de béisbol atrapa una pelota que ha sido bateada (Gigerenzer y Selten 2001). En términos generales, el jugador debe fijar su mirada en la pelota lanzada y correr en su dirección, además debe ajustar la velocidad de modo que el ángulo de su mirada permanezca constante mientras corre. Siguiendo esta heurística, según Gigerenzer y su grupo, un jugador es más eficiente para atrapar una pelota confiando en su ambiente físico y viendo cómo se modifica, que ocupándose en el cálculo formal y la acción que tendría que llevar a cabo para atrapar la pelota.

- Como hemos señalado, los defensores del grupo ABC han apoyado la idea de la racionalidad ecológica (en la que hacen uso de la metáfora de las tijeras) en donde la racionalidad de las heurísticas se define como el acuerdo entre el procesamiento interno y el ambiente. En este caso, según los autores, el ambiente se entiende principalmente como la estructura de información disponible a los agentes. Esta estructura de información está definida por propiedades como la redundancia de las pistas, la variabilidad de validez de las pistas, el tamaño de las muestras, etc. Así, según los defensores de la racionalidad ecológica, la ejecución del razonamiento está determinada por el acuerdo entre las estrategias de razonamiento y los rasgos informacionales del ambiente que implican el éxito relativo

de su proyecto de investigación y no solamente con las tareas de razonamiento que hemos revisado en las secciones anteriores. 
de una estrategia. En ese sentido, la racionalidad ecológica no es algo que dependa de un algoritmo procesado internamente, sino de un "complejo algoritmo-interno-en-un-ambiente-específico" (Arnau et al. 2013, p. 10). Los autores señalan, además, que algunas veces lo normativo no se encuentra simplemente en el ambiente, sino que es producto del acoplamiento en curso del agente con el ambiente. Es relevante tener en cuenta cómo la conducta del agente moldea el ambiente que determina la ejecución de la estrategia de razonamiento disponible. ${ }^{14}$

- Según diversos autores, una de las tareas de las teorías del razonamiento es ofrecer estrategias de cómo se debe razonar; de hecho, los defensores de la racionalidad ecológica han intentado llevar a cabo esta tarea (Bishop y Trout 2005). Tomando en cuenta la metáfora de las tijeras usada por la racionalidad acotada, para lograr un buen ajuste entre las navajas se puede cambiar lo que ocurre tanto en el interior de los agentes como el ambiente mismo. La racionalidad ecológica ha desarrollado las dos estrategias. Un ejemplo de la segunda estrategia es el paso del uso de porcentajes al uso de frecuencias que usan los médicos para evaluar la probabilidad de riesgos. Al igual que el ejemplo de "Linda, la cajera feminista" que hemos expuesto anteriormente, se tienen trabajos de cómo los médicos evalúan la confiabilidad de un diagnóstico médico. En general, cuando la información es presentada en probabilidades subjetivas, los médicos suelen tener una mala ejecución; en cambio, cuando el mismo problema se presenta en frecuencias, la ejecución mejora considerablemente (Gigerenzer y Hoffrage 1995). Lo interesante aquí para los propósitos de este trabajo es que Arnau et al. (2013) mencionan que el ambiente se entiende de manera distinta a como se entienden el ambiente físico y la estructura de la información. No obstante, no aclaran lo anterior y se limitan a señalar que:

el objeto de la manipulación de las propuestas de cambia-el-ambiente no es ni el ambiente material que desempeña un papel en la ejecución de la heurística ni en las propiedades de la estructura de la información del ambiente que desempeña un papel general en la evaluación de la ejecución de la heurística. Más bien, se centra en los recursos simbólicos y representacionales, que por eso mismo se asume que desempeñan un papel computacional. (2013, p. 12, las cursivas son nuestras.)

${ }^{14}$ De hecho, Arnau et al. (2013) defienden que la racionalidad ecológica es un tipo de cognición andamiada, pues además de reconocer la importancia de lo que está fuera del sujeto, admiten que el sujeto puede modificar el ambiente y éste al sujeto. 
Si el recurso simbólico y representacional es el cambio de formato en el que se presenta el problema, entonces, en los casos como "Linda, la cajera feminista" y el diagnóstico médico, Gigerenzer y su grupo conciben el ambiente como un tipo de formato representacional, es decir, la idea de contexto revisada en la sección 2.

En suma, Arnau et al. (2013) consideran que en el trabajo de la racionalidad ecológica existen al menos tres maneras distintas de entender el ambiente, cada una relacionada con distintos aspectos en las teorías de razonamiento, i.e., el descriptivo, el normativo y el prescriptivo. En el aspecto descriptivo se ve al ambiente como el ambiente físico; en el aspecto normativo se concibe como la estructura de información disponible al sujeto y, por último, en el aspecto prescriptivo se concibe el ambiente como los recursos simbólicos y representacionales.

Independientemente de las distinciones anteriores, no deseamos evaluar la propuesta de Arnau et al. (2013), o las críticas hechas a la racionalidad acotada de que su noción de ambiente es ambigua al no distinguir entre el AAE y los ambientes actuales (Stanovich y West 2003), sino que nos limitaremos a usarla para mostrar cómo, si la noción de contexto se entiende como ambiente dentro de la teoría conocida como racionalidad ecológica, entonces existen distintas maneras de entender qué es un contexto.

\section{Discusión}

A pesar de que la noción de contexto se encuentra en diferentes teorías de la psicología cognitiva y de que su importancia ha sido señalada tanto para explicar cómo los seres humanos razonamos como para determinar si razonamos de manera adecuada o no, no existe en la literatura un análisis sistemático dentro de la psicología cognitiva de qué es un contexto. Hemos presentado aquí varias ideas que están detrás de la noción de contexto según distintos psicólogos cognitivos, ${ }^{15}$ mismas que pueden enlistarse de la siguiente manera:

1) Contexto como familiaridad. Como hemos argumentado, la familiaridad se entiende como el contenido de un problema. En diferentes trabajos sobre la tarea de selección de tarjetas se ha observado

${ }^{15}$ Los ejemplos que hemos puesto en las secciones precedentes ilustran cuáles son las ideas a las que hacen referencia los contextos. No es que queramos generalizar a partir de unos cuantos ejemplos para señalar cuáles son las diferentes nociones de contexto en distintas teorías de la psicología cognitiva, sino que los ejemplos nos sirven para mostrar cómo varias teorías psicológicas han usado ese término en tareas de razonamiento concretas. 
que la ejecución de los sujetos cambia dependiendo del contexto entendido como familiaridad y, en ese sentido, del contenido de la tarea en cuestión.

2) Contexto como formato. Se ha expuesto cómo en relación con el razonamiento probabilístico se defiende que puede haber contextos formados por formatos subjetivos o por formatos frecuentistas. A partir del famoso experimento de "Linda, la cajera feminista", en el cual se ha sostenido que los sujetos cometen la falacia de la conjunción, se ha formulado el mismo problema en un contexto diferente de la versión original de dicho experimento. Así, el mismo problema en un contexto diferente, se ha argumentado, hace que los sujetos razonen de manera adecuada. En este caso se interpreta el contexto como el formato en el que el problema se presenta, en especial, se ha sostenido que se puede distinguir entre un formato subjetivo y un formato frecuentista.

3) Contexto como perspectiva. Se ha señalado que los psicólogos evolucionistas aplican la noción de contexto al punto de vista que debe tomar un sujeto dentro de un problema de razonamiento. En la tarea de selección de tarjetas con contenido familiar, se ha observado que la ejecución de los sujetos puede cambiar a partir de la perspectiva que los sujetos asumen dentro de la tarea de razonamiento. Se dice en estos trabajos que los sujetos en las distintas tareas de selección se enfrentan a diferentes contextos, entendiendo aquí el contexto como la perspectiva que el sujeto adopta en dicha tarea.

4) Contexto originado por una estructura lógica distinta. Un contexto puede ser sugerido por un conectivo de tipo indicativo, y otro por el de un conectivo de tipo deóntico. Teniendo como telón de fondo las explicaciones que los psicólogos evolucionistas ofrecen para explicar la buena ejecución en la tarea de selección de tarjetas, se ha señalado que la buena ejecución en tales tareas se debe a que en algunas versiones de dichas tareas se hace uso de un conector lógico de tipo deóntico, mientras que en otras se hace uso de un conector de tipo indicativo. Lo interesante para los propósitos de nuestro trabajo es que los defensores de esta propuesta consideran que el conector, junto con una "historia", hace que lo sujetos se enfrenten a contextos distintos dentro de la tarea de selección de tarjetas.

5) Contexto como ambiente. El grupo ABC ha defendido la idea de que la racionalidad surge de la intersección entre los mecanismos cognitivos y el ambiente. Defendemos que una manera de entender el 
contexto es equiparándolo con la noción de ambiente; sin embargo, a pesar de la importancia que esta noción tiene dentro del grupo $\mathrm{ABC}$, no existe una única manera de concebirla. Frente a lo anterior, existen distintas maneras de entender qué es el ambiente (por un lado, el AAE y el ambiente actual y, por otro lado, los aspectos descriptivo, normativo y prescriptivo del mismo) y, en consecuencia, existen distintas maneras de entender qué sería un contexto.

Como hemos señalado en los primeros párrafos, no es nuestro propósito indicar cuál es la mejor noción de contexto (de hecho, probablemente no existe tal cosa como una mejor noción de contexto), sino mostrar cómo con dicho término hace referencia a distintas ideas. Frente al listado de nociones de contexto que hemos propuesto es posible preguntarnos cuál es la relación entre éstas. A continuación presentamos un esbozo de cuáles podrían ser estas relaciones con respecto a la primera noción de contexto. Hacemos la comparación a partir de la primera noción no porque ésta sea la más fundamental, sino simplemente para mostrar cómo se tendría que hacer una revisión de las distintas nociones. La revisión que haremos será llevada a cabo por pares de nociones, para saber cuáles serían los posibles vínculos entre las nociones aquí expuestas. Reunidas por pares de nociones tenemos lo siguiente:

A) Noción 1 - contexto como familiaridad y noción 2 - contexto como formato del problema. Como hemos señalado, la familiaridad de un problema de razonamiento se puede entender como el contenido del problema. En el caso del formato del problema de razonamiento, éste puede entenderse de modo frecuentista o subjetivista. Si la familiaridad se entiende como contenido, podría sostenerse que no hay una relación necesaria entre el contenido y que el formato sea o bien subjetivista o bien frecuentista. En otras palabras, la noción $1-$ contexto como familiaridad no implica la noción 2 - contexto como formato.

Por otro lado, el tipo de formato no tiene relación alguna con que éste sea familiar o no, aunque se podría sostener que el formato frecuentista permite que sea más claro el contenido del problema; es decir, se podría defender que cuando un problema se encuentra en formato de frecuencia, el contenido es mejor asimilado por los sujetos debido a que se vuelve familiar. Sin embargo, esto no significa que el formato de frecuencia (o el formato subjetivista) sea equivalente a hablar de contenido, aunque dependiendo del formato el contenido puede ser más asequible. Por lo tanto, la noción 2 - contexto como 
formato del problema no implica la noción 1 - contexto como familiaridad.

B) Noción 1 - contexto como familiaridad y noción 3 - contexto como perspectiva. Como hemos señalado, la familiaridad de un problema de razonamiento se puede entender como el contenido del problema. Por su parte, la perspectiva se entiende como el papel del sujeto dentro del problema de razonamiento. Si la familiaridad se entiende como contenido, ésta no implica la noción de perspectiva, puesto que el contenido de un problema es indiferente al papel que el sujeto tome en el problema. Si lo anterior es correcto, entonces la noción 1 - contexto como familiaridad no implica la noción 3 contexto como perspectiva.

No obstante, si por contexto entendemos sólo el papel del sujeto dentro de un problema de razonamiento, éste es indiferente al contenido del mismo. Esto es, la perspectiva que un sujeto tiene dentro de un problema no nos dice nada sobre el tipo de material del problema; en el caso de la tarea de selección de tarjetas, por ejemplo, el papel del sujeto puede ser indiferente a si el problema implica medios de transporte y lugares que él nunca haya usado o nunca haya visitado. En otras palabras, la perspectiva del sujeto parece neutral con respecto al contenido del problema mismo. En suma, parece que la noción 3 - contexto como perspectiva es neutral a la noción 1 - contexto como familiaridad.

C) Noción 1 - contexto como familiaridad y noción 4-contexto como estructura lógica distinta. La familiaridad de un problema de razonamiento se puede entender como el contenido del problema. Por su parte, la estructura lógica de un problema de razonamiento puede verse como la naturaleza del conectivo lógico, en particular si es de tipo indicativo o deóntico. Si la familiaridad se entiende como contenido, éste puede ser indiferente a que el conectivo sea de tipo indicativo o deóntico. Lo anterior se debe a que, por ejemplo, en el caso de la tarea de selección de tarjetas lo que cada una de las tarjetas contenga no afecta al condicional que esté en juego. Por tal motivo, la noción 1 - contexto como familiaridad es indiferente a la noción 4 - contexto como estructura lógica distinta.

Si partimos de la idea de contexto como estructura lógica distinta, éste no nos dice nada acerca del contenido mismo de una tarea de razonamiento. De nuevo, trayendo a colación la tarea de selección de tarjetas, el contenido puede ser familiar (hablando de objetos familiares como alimentos o medios de transporte) inscrito en condiciona- 
les indicativos o deónticos. No obstante, los teóricos que han enfatizado el papel del conectivo lógico han señalado que la "historia" puede ayudar a identificar el tipo de conectivo lógico del problema. Si, como señalamos, la historia puede entenderse como contenido, entonces se podría argumentar a favor de un traslape entre contexto como conectivo e historia, y la noción de contexto como familiaridad. Por lo anterior, la noción de contexto 4 - contexto como estructura lógica distinta se traslapa con la noción 1 - contexto como familiaridad.

D) Noción 1 - contexto como familiaridad y noción 5 - contexto como ambiente. Una vez más, se ha dicho que cuando el contexto hace referencia a la familiaridad de un problema, éste puede consistir en el contenido. En cuanto al contexto como ambiente hemos sostenido que éste puede hacer referencia al AAE o al ambiente actual, además puede entenderse desde una manera descriptiva, normativa o prescriptiva. Cuando los psicólogos evolucionistas hacen uso de la familiaridad no queda del todo claro que hagan referencia al contenido con el que actualmente se enfrentan los seres humanos modernos o aquel al que se enfrentaron en el AAE. De hecho, la crítica de Stanovich y West (2003) a la noción de ambiente en Gigerenzer y su grupo, es la misma que aplican a los psicólogos evolucionistas. Si éste es el caso, se puede decir que la noción de contexto como familiaridad en los psicólogos evolucionistas al menos podría ser leída de las dos maneras, lo cual es compatible con la noción de contexto como ambiente (ya sea como ambiente actual o como AAE). Sin embargo, si además se tiene en mente que la noción de contexto como ambiente podría ser entendida en sentido descriptivo, normativo y prescriptivo, cabe preguntarse: ¿la noción de contexto como familiaridad supone estos tres aspectos? Si traemos a colación la tarea de selección de tarjetas, habitualmente cuando los psicólogos evolucionistas piensan en que el caso de la raíz de cassava no les es familiar a los sujetos, ellos parecen referirse a que los sujetos de hecho no se encuentran inmersos en tales contextos. Eso nos lleva a pensar que en general los psicólogos evolucionistas están concibiendo la familiaridad de manera descriptiva y no como las normas o las prescripciones que el sujeto debe seguir. Si esto es correcto, el contexto como familiaridad puede ser compatible con el contexto como ambiente sólo en cuanto pueda verse como la familiaridad del AAE y la familiaridad actual. Además, el contexto como familiaridad puede verse en un sentido descriptivo, pero no queda claro que los psicólogos evolucionistas lo entiendan de manera normativa o prescriptiva como lo ha hecho Gigerenzer, por 
lo cual la noción 1 - contexto como familiaridad no podría abarcar todos los aspectos de la noción 5 - contexto como ambiente.

Si el contexto se concibe como ambiente, ¿se sigue de ahí que pueda entenderse como familiaridad y en qué sentido? Como se señaló en el párrafo anterior, se ha criticado que el grupo ABC y los psicólogos evolucionistas no dicen claramente si el contexto hace referencia al AAE o al ambiente actual, motivo por el cual creemos que la relación entre contexto como ambiente y contexto como familiaridad son compatibles. Lo que no es tan claro es si el ambiente en sus aspectos descriptivo, normativo y prescriptivo es compatible o no con la noción de contexto como familiaridad. Si como parece ser el caso, la familiaridad se entiende por los psicólogos evolucionistas sólo en su aspecto descriptivo, entonces la noción 5 - contexto como ambiente sí implicaría la noción 1 - contexto como familiaridad, pero es más amplia que éste abarcando los ámbitos normativo y prescriptivo.

Creemos que el análisis anterior no es sino un primer intento de entender cuáles son las relaciones entre las nociones de contexto que se encuentran implícitas en la literatura. Un análisis más detallado se encuentra más allá de los alcances de este trabajo; no obstante, del análisis anterior tenemos lo siguiente:

i. La noción 1 - contexto como familiaridad no implica la noción 2 - contexto como formato.

ii. La noción 2 - contexto como formato frecuentista o subjetivista no implica la noción 1 - contexto como familiaridad.

iii. La noción 1 - contexto como familiaridad no implica la noción 3 - contexto como perspectiva.

iv. La noción 3 - contexto como perspectiva es neutral a la noción 1 - contexto como familiaridad.

v. La noción 1 - contexto como familiaridad es indiferente a la noción 4 - contexto como estructura lógica distinta.

vi. La noción 4 - contexto como estructura lógica distinta se traslapa con la noción 1 - contexto como familiaridad.

vii. La noción 1 - contexto como familiaridad no podría abarcar todos los aspectos de la noción 5 - contexto como ambiente. Sin embargo, estas dos nociones de contexto comparten su ambigüedad con respecto a si contexto hace referencia al AAE o al ambiente actual. 
viii. La noción 5 - contexto como ambiente sí implicaría la noción de contexto 1 - contexto como familiaridad, pero es más amplia que éste por abarcar los ámbitos normativo y prescriptivo.

Si lo anteriormente presentado es correcto, entonces nos damos cuenta de que en la psicología cognitiva del razonamiento algunas veces existen coincidencias y traslapes entre los contextos, mientras que otras veces parece que hacen referencia a algo muy distinto. Por ejemplo, en el caso (viii) existen traslapes en qué se está entendiendo por un contexto; mientras que en el caso (iii) parece no haber un vínculo entre estos dos modos de caracterizar un contexto.

Una vez que se ha hecho la comparación entre la noción de contexto 1 y el resto de nociones, surge la pregunta de si podríamos hacer la comparación de la noción 2 y así sucesivamente. Lo anterior, por supuesto, se puede hacer, pero no creemos que sea razonable para la extensión de un artículo. Como hemos señalado anteriormente, comparar la noción 1 con el resto de nociones se hace por una cuestión pragmática y no porque dicha noción sea más básica o elemental que las otras nociones de contexto. Hacemos la relación para mostrar cómo puede haber coincidencias y traslapes algunas veces, y cómo otras veces puede haber distintos referentes entre las nociones de contexto. Hecho esto, si con la comparación entre la acepción (1) es posible mostrar lo anterior, ello vuelve innecesario comparar las siguientes acepciones.

Antes de concluir este trabajo queremos abordar dos cuestionamientos con los que queremos sugerir hacia dónde se puede ir con el análisis de las diferentes nociones que hemos presentado. El primer cuestionamiento es acerca de dónde proviene la diferencia entre las nociones de contexto que aquí hemos presentado. Por su parte, el segundo cuestionamiento es sobre qué consecuencias tendrían estas distinciones para el debate sobre el razonamiento y la racionalidad. Dada la extensión del análisis de las diferentes nociones de contexto, sólo esbozaremos los lineamientos generales de las respuestas a estos dos cuestionamientos. Veamos.

En cuanto a la primera pregunta, esto es, ¿a qué se debe que haya diferentes nociones de contexto?, o ¿̨de dónde vienen o cómo se originan dichas diferencias?, habría diferentes maneras de contestar esta pregunta. Una de ellas sería que es posible que las diferentes nociones de contexto que hemos enlistado se deban a que cada teoría del razonamiento parte de supuestos teóricos distintos y que, por ende, la noción de contexto esté - empleando una expresión muy usada en filosofía de la ciencia - "cargada de teoría". Si esto es así, 
la noción de contexto cambia, por ejemplo, cuando alguien defiende la psicología evolucionista o cuando alguien más defiende la racionalidad acotada, a partir de los supuestos teóricos que cada una de estas teorías tiene. Esta respuesta podría apoyarse en que una de las nociones de contexto, en particular la noción 2, está apoyada por la psicología evolucionista, mientras que la noción 5 está apoyada por la racionalidad ecológica. Sin embargo, antes de analizar por qué estas teorías defienden estas nociones, es posible percatarse de que el problema, con la sugerencia de que las nociones de contexto son diferentes por tratarse de diferentes teorías, es que un mismo autor o los defensores de una misma teoría - por ejemplo, la psicología evolucionista (véanse Cosmides y Tooby 1992 y 1996) - a veces usan el término contexto como la familiaridad del problema (es decir, como la noción 1) y otras veces como formato (es decir, como la noción 2). Esto nos hace pensar que no es el caso que los principios que los psicólogos evolucionistas ven como centrales para su propuesta o su metodología (véase Cosmides y Tooby 1997) determinen el modo en que deben entender el contexto, lo cual sugiere otra respuesta al cuestionamiento que nos hemos planteado.

Otra sugerencia es que la noción de contexto es simplemente polisémica y que los psicólogos la han usado para englobar fenómenos sobre los cuales no se ha reflexionado lo suficiente. Entre los fenómenos en los que se ha aplicado están aquellos que se descubrieron cambiando ciertas variables dentro de los experimentos de razonamiento ofrecidos, por ejemplo, históricamente se descubrió que en el caso de la tarea de selección de tarjetas la familiaridad del contenido de un problema tendía a tener resultados diferentes en la ejecución y a ese contenido se le denominó el contexto del problema. En otros casos, como hemos mostrado, se descubrió que la ejecución cambiaba si la perspectiva del sujeto en una tarea de selección cambiaba y a eso se llamó también contexto. De hecho, el uso coloquial del término "contexto" se ocupa para referirse a ese tipo de elementos y parece que en realidad los psicólogos cognitivos hicieron uso de ese término sin haber reflexionado lo suficiente sobre él. Un problema con esta sugerencia es que hacer uso de un término cotidiano y la ausencia de discusión sobre el mismo podría crear la visión de que no hay nada que discutir. Sin embargo, lo anterior es un error, ya que, por un lado, al principio de este trabajo hemos mostrado cómo en una de las discusiones más importantes en la psicología cognitiva dos grupos de psicólogos se acusan mutuamente de no tomar en cuenta el contexto; por otro lado, hemos mostrado a lo largo de este trabajo que la noción de contexto hace referencia a diferentes ideas, no todas ellas 
compatibles, y que el diálogo o discusión entre las teorías tendría que tener en cuenta que los términos usados tienen un significado diferente.

Una tercera manera de abordar el primer cuestionamiento sería argumentar que los diferentes usos de contexto tienen su origen en el uso de lógicas diferentes o en una variedad de mecanismos cognitivos que pueden guiar los juicios en diferentes situaciones. Una propuesta interesante al respecto es la que defienden Stenning y van Lambalgen (2008). Estos autores sostienen que las diferentes nociones de contexto tienen que ver con diferentes interpretaciones de los condicionales y sobre todo con la distinción que ellos hacen entre razonar hacia una interpretación y razonar desde una interpretación. Razonar hacia una interpretación es lo que se supone debe pasar en una tarea típica de inferencia; a saber, dadas ciertas premisas se determina si se sigue una conclusión dada. Sin embargo, debido a que las premisas están formuladas en lenguaje natural, se abre el espacio para diferentes interpretaciones lógicas de la tarea dada (razonar desde una interpretación) (Stenning y van Lambalgen 2008, p. 50). Así, si tomamos en consideración esta propuesta, las diferentes nociones de contexto podrían ser explicadas debido a diferentes atribuciones de forma lógica y no simplemente por distintas interpretaciones de la forma lógica de la oración. En otras palabras, la noción de contexto estaría fuertemente relacionada con procesos interpretativos que surgen de cuestiones semánticas en las tareas de razonamiento. Creemos que esta propuesta es interesante y podría explicar el origen de algunas nociones de contexto que hemos presentado en este trabajo; por ejemplo, aquellas que involucran la estructura lógica de los problemas. No obstante, esta propuesta no abarca todas las nociones de contexto presentadas aquí. Por ejemplo, no queda claro cómo la distinción entre razonar hacia una interpretación y razonar desde una interpretación puede explicar la concepción de contexto como ambiente.

Independientemente de que a la pregunta de a qué se debe que haya diferentes nociones de contexto se responda que a la carga teórica de la observación, a la polisemia y descuido del uso de esa noción, o a distinciones como la que Stenning y van Lambalgen defienden, consideramos que distintos psicólogos cognitivos presuponen que la noción de contexto se usa de la misma manera y que en ese sentido es un término neutral, no problemático e independiente de las teorías que lo usan o de su interpretación lógica. Como en el debate presentado en los primeros párrafos, es suponiendo que se usa del mismo modo el término como se hace posible ver a los defensores de la racionalidad ecológica acusando a los defensores de la tradición de 
heurística y sesgo de no tomar en cuenta el contexto, cuando los defensores de esta última han acusado a los primeros de malinterpretar su trabajo. Esto nos lleva a la segunda pregunta que habíamos planteado, esto es, cuáles son las consecuencias de no distinguir entre contextos, o bien, cuáles podrían ser las consecuencias de hacer la distinción entre contextos para los debates de razonamiento y racionalidad.

Uno de los motivos por los que la noción de contexto es importante es que, como se ha visto en las cinco nociones que hemos presentado, ellas se encuentran ligadas a la cuestión de si se resuelve una tarea de razonamiento de manera adecuada o no. Sea que el contexto se entienda como familiaridad, como perspectiva, como formato, como estructura lógica o como ambiente, nos damos cuenta de que estas maneras de entenderlo están ligadas a que si el contexto cambia, se puede tener una ejecución correcta de razonamiento. Si esto es así, la noción de contexto está vinculada fuertemente a la noción de racionalidad, ${ }^{16}$ esto es, al aspecto que evalúa si el sujeto razona de manera adecuada o no, o, para ponerlo de otro modo, si el sujeto sigue las normas adecuadas del buen razonamiento. En otras palabras, la noción de contexto, o sus diferentes maneras de entenderlo, conecta cómo los sujetos razonan y cómo deben razonar. De hecho, en la noción 5, esto es, contexto como ambiente, se dice algo todavía más fuerte, viz., que el contexto en cierto modo determina qué significa razonar correctamente. Si la noción de contexto es tan importante para la cuestión de la racionalidad, entonces es posible percatarse de cuán importante es el análisis de dicha noción, puesto que pensar que hay un único referente de lo que es un contexto o confundir las diferentes ideas a las que la noción de contexto refiere, podría generar debates acerca de si los sujetos razonamos de manera adecuada o no.

Un ejemplo particular de ese tipo de debates es aquel que se desarrolla entre la tradición de heurística y sesgo y el grupo ABC. Los defensores de estos programas tienen visiones distintas acerca de la racionalidad, viz., la tradición de heurística y sesgo se asocia a la visión de que no razonamos de manera adecuada, mientras que

\footnotetext{
${ }^{16}$ La relación entre las nociones de contexto y el debate sobre la racionalidad se puede ver en que en algunas nociones de contexto de esta discusión parecen asumirse visiones normativas diferentes. En otras palabras, en algunas nociones de contexto se evalúa lo que es el buen razonamiento según distintas visiones de la racionalidad. Por ejemplo, en las nociones de contexto 1 y 4 se asume una teoría de la racionalidad, a saber, la visión estándar de la racionalidad (véase la nota 4 en este texto), distinta a la que se asume en la noción 5, esto es, una racionalidad instrumental.
} 
el grupo $\mathrm{ABC}$ tiene una visión optimista en la que, en términos generales, razonamos de manera adecuada. Como hemos señalado en los párrafos introductorios, la tradición de heurística y sesgo, y el grupo ABC parecen acusarse mutuamente de no tomar en cuenta el contexto. ¿Cómo se relaciona esto con el análisis sobre contexto que hemos realizado? Kahneman y Tversky (1996, p. 583) señalan que el grupo $\mathrm{ABC}$ considera que los errores de razonamiento pueden cambiar si se manipula el formato en que se presentan los problemas; además, al mismo tiempo señalan que los procesos mentales dependen del contenido y las relaciones. Si las ideas anteriores se conectan con las nociones de contexto que hemos defendido, teniendo en cuenta que Kahneman y Tversky señalan que el contexto es algo que sirve para entender cómo razonamos, entonces es posible que estén pensando que ellos toman en cuenta el contexto porque de hecho toman en cuenta el contenido, es decir, están pensado en la noción 1 de contexto que nosotros hemos señalado; mientras que no les parece adecuada la acusación de Gigerenzer, pues ellos no identifican al formato con el contexto, como de hecho es la manera en que parece que Gigerenzer y el grupo $\mathrm{ABC}$ la interpretan. Como argumentamos, según nuestro análisis, la postura de Gigerenzer corresponde a la noción 2 de contexto, esto es, contexto como formato.

Otra manera interesante de ver por qué es importante analizar la noción de contexto en este debate sería tomar en consideración cómo ambas teorías ven la relación entre contexto y racionalidad. En este sentido, "el desacuerdo" entre estas teorías podría ser "normativo" (Kahneman y Tversky 1996, p. 589). Gigerenzer sostiene que para evaluar lo que cuenta como buen razonamiento se debe investigar primero el contexto de una tarea de razonamiento y a partir de ahí inferir la teoría normativa que se ha de utilizar. Por el contrario, Kahneman y Tversky, según Gigerenzer, en su evaluación del buen razonamiento empiezan con una teoría normativa particular (en especial con reglas estadísticas) sin tomar en cuenta el contexto de la tarea por resolver. En otras palabras, para Gigerenzer el contexto determina la teoría normativa que se debe utilizar para evaluar el buen razonamiento, mientras que para Kahneman y Tversky la teoría normativa debe establecer lo que cuenta como contexto en una tarea de razonamiento.

Mucho de lo que se ha explorado en la literatura sobre la discusión entre estos grupos de psicólogos gira en torno a si comparten el mismo estándar de racionalidad, o si comparten la misma descripción del 
razonamiento; pero no se ha explorado que, además de esos elementos, no parecen compartir la manera de entender qué es un contexto y cómo la modificación de éste puede cambiar el modo en que los seres humanos razonamos. Si lo esbozado a lo largo de este trabajo es correcto, es posible ver cómo la noción de contexto y su análisis es útil para reinterpretar o agregar otros elementos a los debates en torno al razonamiento y la racionalidad, en particular aquel entre la tradición de heurística y sesgo y el grupo ABC. Lo anterior es una de las consecuencias que habría al hacer las distinciones entre contextos que hemos realizado en este trabajo.

\section{Conclusiones}

En este texto hemos defendido que en la literatura de la psicología del razonamiento no existe una única caracterización de qué es un contexto de razonamiento. Analizando la literatura hemos sostenido que existen al menos cinco ideas detrás de la noción de contexto: 1) contexto como familiaridad, 2) contexto como formato, 3) contexto como perspectiva, 4) contexto como estructura lógica distinta y 5) contexto como ambiente.

A partir de la lista anterior podemos sostener que la noción de contexto en psicología cognitiva no hace referencia a una única idea. Pero basándonos en esta conclusión, que parece natural a partir de la literatura sobre teorías cognitivas del razonamiento, hemos propuesto posibles maneras de relacionar en pares las cinco nociones aquí presentadas mostrando cómo hay casos en donde puede haber traslapes. No obstante, hay otros casos en donde no queda claro que el término contexto haga referencia a la misma idea. La noción de contexto de razonamiento ha desempeñado un papel importante para explicar cómo es que los seres humanos razonamos y si lo hacemos de manera adecuada o no; pero es necesario un análisis más profundo partiendo de que, como hemos argumentado en este trabajo, en la literatura psicológica no se hace referencia a una única idea con dicha noción. La clarificación que proponemos en este trabajo puede ayudar a entender debates particulares en la psicología del razonamiento, como el de la tradición de heurística y sesgo con el grupo ABC, ya que dicho debate en parte gira en torno a si cada teoría toma en cuenta o no el contexto en que razona el sujeto (Kahneman y Tversky 1996, Gigerenzer 1996). 


\section{BIBLIOGRAFÍA}

Arnau, E., S. Ayala y T. Sturm, 2013, "Cognitive Externalism Meets Bounded Rationality", Philosophical Psychology, vol. 27, no. 1, pp. 5064.

Bishop, M. y J.D.Trout, 2005, Epistemology and the Psychology of Human Judgment, Oxford University Press, Nueva York.

Bolhuis, J., G. Brown, R. Richardson y K. Laland, 2011, "Darwin in Mind: New Opportunities for Evolutionary Psychology", PLOS Biology, vol. 9, no. 7 , pp. 1-8.

Buller, D., 2005, Adapting Minds. Evolutionary Psychology and the Persistent Quest for Human Nature, The MIT Press, Cambridge, Mass.

Buller, D., J. Fodor y T. Crume, 2005, "The Emperor Is Still UnderDressed", Trends in Cognitive Science, vol. 9, no. 11, pp. 508-510.

Chiappe, D. y R. Gardner, 2011, "The Modularity Debate in Evolutionary Psychology", Theory \& Psychology, vol. 22, no. 5, pp. 669-682.

Cosmides, L. y J. Tooby, 2005, "Neurocognitive Adaptations Designed for Social Exchange", en D. Buss (ed.), Evolutionary Psychology Handbook, Willer, Nueva York, pp. 584-627.

—_, 1997, "Evolutionary Psychology: A Primer", Center for Evolutionary Psychology, University of California, Santa Barbara; disponible en: $<$ https://www.cep.ucsb.edu/primer.html $>$.

— 1996, "Are Humans Good Intuitive Statisticians after All? Rethinking Some Conclusions from the Literature on Judgment under Certainty", Cognition, vol. 58, no. 1, pp. 1-73.

__ 1992, "Cognitive Adaptations for Social Exchange", en J. Barkow et al. (eds.), The Adapted Mind, Oxford University Press, Nueva York, pp. 164-228.

_ , 1991, "Reasoning and Natural Selection", Encyclopedia of Human Biology, vol. 6, pp. 493-503.

Fodor, J., 2000, "Why We Are So Good at Catching Cheaters", Cognition, vol. 75 , pp. 29-32.

Gigerenzer, G., 1996, "On Narrow Norms and Vague Heuristics: A Reply to Kahneman and Tversky", Psychological Review, vol. 103, no. 3, pp. $592-598$.

_, 1991, "How to Make Cognitive Illusions Disappear: Beyond 'Heuristics and Biases'", European Review of Social Psychology, vol. 2, pp. 83115.

Gigerenzer, G., P. Todd y Grupo ABC, 1999, Simple Heuristics That Make Us Smart, Oxford University Press, Nueva York.

Gigerenzer, G. y K. Hug, 1992, "Domain-Specific Reasoning: Social Contracts, Cheating and Perspective Change", Cognition, vol. 43, pp. 127171.

Gigerenzer, G. y R. Selten (eds.), 2001, Bounded Rationality. The Adaptive Toolbox, The MIT Press, Cambridge, Mass. 
Gigerenzer, G. y T. Sturm, 2012, "How (Far) Can Rationality Be Naturalized?", Synthese, vol. 187, no. 1, pp. 243-268.

Gigerenzer, G. y U. Hoffrage, 1995, "How to Improve Bayesian Reasoning without Instruction: Frequency Formats", Psychological Review, vol. 102, no. 4, pp. 684-704.

Hertwig, R. y G. Gigerenzer, 1999, "The 'Conjunction Fallacy' Revisited: How Intelligent Inferences Look Like Reasoning Errors", Journal of Behavioral Decision Making, vol. 12, pp. 275-305.

Kahneman, D. y A. Tversky, 1996, "On the Reality of Cognitive Illusions", Psychological Review, vol. 103, no. 1, pp. 582-591.

Kahneman, D., P. Slovic y A. Tversky, 1982, Judgment under Uncertainty: Heuristics and Biases, Cambridge University Press, Cambridge.

Piatelli-Palmarini, M., 2005, Los túneles de la mente, Crítica, Barcelona.

Samuels, R., S. Stich y L. Faucher, 2004, "Reason and Rationality", en I. Niiniluoto et al. (eds.), Handbook of Epistemology, Kluwer, Dordrecht, pp. 131-179.

Stanovich, K. y R. West, 2003, "Evolutionary Versus Instrumental Goals: How Evolutionary Psychology Misconceives Human Rationality", en D. Over (ed.), Evolution and the Psychology of Thinking, Psychology Press, East Sussex, pp. 171-230.

Stein, E., 1996, Without Good Reason. The Rationality Debate in Philosophy and Cognitive Science, Clarendon Press, Oxford.

Stenning, K. y M. van Lambalgen, 2008, Human Reasoning and Cognitive Science, The MIT Press, Cambridge, Mass.

Sturm, T., 2012, “The 'Rationality Wars' in Psychology: Where They Are and Where They Could Go", Inquiry, vol. 55, no. 1, pp. 66-81.

Wason, P.C., 1966, "Reasoning", en B.M. Foss (ed.), New Horizons in Psychology: I, Penguing, Harmondsworth, pp. 106-137.

Recibido el 13 de febrero de 2018; revisado el 29 de mayo de 2019; aceptado el 12 de junio de 2019. 